\title{
FIGO Stage III
}

National Cancer Institute

\section{Source}

National Cancer Institute. FIGO Stage III. NCI Thesaurus. Code C96255.

A FIGO stage term that applies to gynecologic cancers. For cervical cancer, it refers to cancer that extends to the pelvic wall, and/or involves the lower third of vagina, and/or causes hydronephrosis or non-functioning kidney; for endometrial cancer, FIGO stage III is subdivided into stages IIIA and IIIB; in FIGO stage IIIA, there is involvement of the serosa and/or the adnexa; for FIGO stage IIIB, there is vaginal or parametrial involvement. 